ORIGINAL ARTICLE

\title{
Association of chronic fatigue syndrome with human leucocyte antigen class II alleles
}

\author{
J Smith, E L Fritz, J R Kerr, A J Cleare, S Wessely, D L Mattey
}

J Clin Pathol 2005;58:860-863. doi: 10.1136/jcp.2004.022681

See end of article for authors' affiliations

.....................

Correspondence to: Dr J R Kerr, Department of Paediatric Infectious Diseases, St Mary's Campus, Imperial College London, Norfolk Place, London W2 IPG, UK: i.kerr@imperial.ac.uk

Accepted for publication 23 February 2005
Background: A genetic component to the development of chronic fatigue syndrome (CFS) has been proposed, and a possible association between human leucocyte antigen (HLA) class II antigens and chronic fatigue immune dysfunction has been shown in some, but not all, studies.

Aims: To investigate the role of HLA class II antigens in CFS.

Methods: Forty nine patients with CFS were genotyped for the HLA-DRB1, HLA-DQA1, and HLA-DQB1 alleles and the frequency of these alleles was compared with a control group comprising 102 normal individuals from the UK. All patients and controls were from the same region of England and, apart from two patients, were white.

Results: Analysis by $2 \times 2$ contingency tables revealed an increased frequency of HLA-DQA $1{ }^{*} 01$ alleles in patients with CFS $(51.0 \%$ v 35\%; odds ratio (OR), 1.93; $p=0.008)$. HLA-DQB $1{ }^{*} 06$ was also increased in the patients with CFS (30.2\% v 20.0\%; OR, 1.73, $p=0.052)$. Only the association between HLADQA $1^{*} 01$ and CFS was significant in logistic regression models containing HLA-DQA ${ }^{*} 01$ and HLA$D R Q B 1{ }^{*} 06$, and this was independent of HLA-DRB1 alleles. There was a decreased expression of HLA$\mathrm{DRB} 1^{*} 11$ in CFS, although this association disappeared after correction for multiple comparisons.

Conclusions: CFS may be associated with $\mathrm{HLA}-\mathrm{DQA} 1^{*} 01$, although a role for other genes in linkage disequilibrium cannot be ruled out.

\footnotetext{
C
} hronic fatigue syndrome (CFS) is a complex illness that affects approximately $0.2-0.7 \%$ of people in the West. It affects more women than men with an onset usually between 30 and 40 years of age. ${ }^{12}$ The disease manifests as severe incapacitating fatigue that persists for six months or longer, which is not the result of exertion and is not improved by bed rest. Other symptoms include: lack of concentration and/or impaired memory, recurrent sore throats, tender cervical or axillary lymph nodes, muscle pain, new headaches, unrefreshing sleep, and post-exertion malaise. ${ }^{3}$ Diagnosis is made according to the symptom based CFS case definition of the Centers for Disease Control (CDC). ${ }^{4}$

"Lymphocyte proliferation and the production of cytokines and high affinity antibodies is mediated by the HLA system"

The cause of CFS remains largely unknown. Current research using molecular biological techniques has improved our knowledge of the disease. It has been shown that certain viruses-for example, Epstein-Barr virus, cytomegalovirus, ${ }^{5}$ and parvovirus $\mathrm{B} 19^{6}$ - may play an important role in some cases of CFS. Other studies ${ }^{7-11}$ suggest that host immune dysfunction may influence the course of the disease, although one study failed to find evidence of immune dysfunction or immune activation in CFS. ${ }^{12}$ Such differences may depend on which immunological parameters are measured. The immunology of CFS has recently been reviewed by Lyall and colleagues. ${ }^{13}$ Studies on twins ${ }^{14-17}$ and an association with HLA-DR4 ${ }^{7}$ indicate a possible genetic predisposition to the disease. However, some studies have failed to find an association between HLA class II alleles and CFS, ${ }^{18}{ }^{19}$ and one study has suggested a negative association with HLA-DR4. ${ }^{20}$

HLA genes are located in a highly polymorphic locus on chromosome 6 p21, where they encode a family of cell surface glycoproteins responsible for specific antigen presentation and immune activation of other cells. ${ }^{21}$ Lymphocyte proliferation and the production of cytokines and high affinity antibodies is mediated by the HLA system, and various HLA alleles have been associated with both infectious disease and autoimmune dysfunction. ${ }^{22}$ A role for the HLA system in CFS is suggested by the finding of increased concentrations of some cytokines in CFS. ${ }^{63}{ }^{24}$ The possibility of an infective agent in this disease would also indicate involvement of the HLA system. In a recent study ${ }^{25}$ investigating the association between HLA and symptomatic parvovirus B19 infection, we reported that some of the patients fulfilled the criteria for CFS.

The genetic component to the development of CFS is the subject of ongoing research in various laboratories, including our own. This research includes studies on differential gene expression, which may highlight specific genes that are crucial in the disease process. For an improved understanding of the pathophysiology of CFS, in our present study we have further investigated the association between specific HLA class II alleles and the disease. We HLA typed patients who fulfilled the CDC criteria for CFS and compared the results with those for healthy controls.

\section{MATERIALS AND METHODS}

Forty nine patients were diagnosed according to the CDC criteria for chronic fatigue syndrome. ${ }^{4}$ These were referrals to the King's College Hospital CFS Service and came from the South East of England. The ages of the patients ranged from 21 to 63 years, with a mean of 42 years. The female to male ratio was approximately $3: 1$. All patients were white except for two Afro-Caribbeans. Twenty four were depressed and 25 were not depressed, according to an interview by a

Abbreviations: $\mathrm{CFS}$, chronic fatigue syndrome; $\mathrm{Cl}$, confidence interval; HLA, human leucocyte antigen; CDC, Centers for Disease Control; OR, odds ratio 
Table 1 Allele frequencies of HLA-DRB1, HLA-DQA1, and HLA-DQB1 in patients with CFS and controls

\begin{tabular}{|c|c|c|c|c|}
\hline & $\begin{array}{l}\text { CFS } \\
(2 n=98)\end{array}$ & $\begin{array}{l}\text { Allele } \\
\text { frequency }\end{array}$ & $\begin{array}{l}\text { Controls } \\
(2 n=200)\end{array}$ & $\begin{array}{l}\text { Allele } \\
\text { frequency }\end{array}$ \\
\hline \multicolumn{5}{|c|}{ HLA-DRB 1} \\
\hline 01 & 16 & 0.163 & 18 & 0.090 \\
\hline 15 & 14 & 0.143 & 20 & 0.100 \\
\hline 16 & 1 & 0.010 & 4 & 0.020 \\
\hline 03 & 12 & 0.122 & 31 & 0.155 \\
\hline 04 & 13 & 0.133 & 41 & 0.205 \\
\hline 11 & 5 & $0.051^{*}$ & 25 & 0.125 \\
\hline 12 & 1 & 0.010 & 0 & 0.000 \\
\hline 13 & 16 & 0.163 & 22 & 0.110 \\
\hline 14 & 4 & 0.041 & 5 & 0.025 \\
\hline 07 & 12 & 0.122 & 26 & 0.130 \\
\hline 08 & 3 & 0.031 & 3 & 0.015 \\
\hline 09 & 1 & 0.010 & 2 & 0.015 \\
\hline 10 & 0 & 0.010 & 2 & 0.010 \\
\hline \multicolumn{5}{|c|}{ HLA-DQA] } \\
\hline 01 & 50 & $0.510 \dagger$ & 70 & 0.350 \\
\hline 02 & 12 & 0.122 & 29 & 0.145 \\
\hline 03 & 15 & 0.153 & 43 & 0.215 \\
\hline 04 & 2 & 0.020 & 3 & 0.015 \\
\hline 05 & 19 & 0.194 & 55 & 0.275 \\
\hline \multicolumn{5}{|c|}{ HLA-DQB 1} \\
\hline 02 & 20 & 0.208 & 50 & 0.250 \\
\hline 0301 & 11 & 0.115 & 43 & 0.215 \\
\hline 0302 & 8 & 0.083 & 24 & 0.120 \\
\hline 0303 & 5 & 0.052 & 10 & 0.050 \\
\hline 04 & 2 & 0.021 & 3 & 0.015 \\
\hline 05 & 21 & 0.219 & 30 & 0.150 \\
\hline 06 & 29 & $0.302 \ddagger$ & 40 & 0.200 \\
\hline
\end{tabular}

The frequency distribution of the alleles for each of the HLA class II markers in the CFS group was compared with that of controls by $\chi^{2}$ tests for homogeneity. No overall difference was found in the frequency distribution of the HLA-DRB1, HLA-DQA1, or HLA-DQB1 alleles in the CFS group compared with controls.

Individual alleles in the CFS group that showed significant differences compared with the controls (by examination of adjusted residuals) were examined further by $\chi^{2}$ tests using $2 \times 2$ contingency tables.

All comparisons versus controls: ${ }^{*} \mathrm{OR}, 0.40 ; 95 \% \mathrm{Cl}, 0.12$ to 1.08 ; $\mathrm{p}=0.046 ;+O R, 1.93 ; 95 \% \mathrm{Cl}, 1.2$ to $3.3 ; \mathrm{p}=0.008 ; \pm O R, 1.73 ; 95 \%$ $\mathrm{Cl}, 0.96$ to $3.1 ; \mathrm{p}=0.052$.

CFS, chronic fatigue syndrome; $\mathrm{Cl}$, confidence interval; HLA, human leucocyte antigen; $O R$, odds ratio.

psychiatrist using a semi-structured interview format and DSM-IV. ${ }^{26}$

\section{Controls}

The controls were a group of 102 white cadaveric donors whose cause of death was trauma based and who had no reported symptoms of either fatigue or CFS.

\section{HLA typing}

Patients and controls were typed for HLA class II alleles: HLA-DRB1, HLA-DQAl, and HLA-DQBl by polymerase chain reaction using sequence specific primers, with DNA extracted from whole blood.

\section{Statistical analysis}

The frequency distribution of alleles for HLA-DRB1, HLADQAl, and HLA-DQB I in patients with CFS was initially compared with that of controls by $\chi^{2}$ tests for homogeneity. The alleles that contributed most to the $\chi^{2}$ were determined by examining the adjusted residuals (deviations from expected values). Each allele in the disease group that showed a significant difference compared with the controls was then examined further by either $\chi^{2}$ or Fisher exact analysis of $2 \times 2$ contingency tables. The strength of the association between CFS and the HLA alleles and phenotypes was estimated using odds ratios (OR) and 95\% confidence intervals (CI). To examine whether any association of HLA class II alleles was independent of other alleles in linkage disequilibrium we carried out multivariate logistic regression analyses with different combinations of HLA-DRBl, HLADQAl, and HLA-DQB1 phenotypes as independent (explanatory) variables, and the presence or absence of CFS as the dependent (outcome) variable. Analyses were carried out using the Number Cruncher Statistical Package for Windows (NCSS 2000), or the PEPI software package ( $v$ 2.0) for epidemiological analysis.

\section{RESULTS}

\section{HLA-DRB 1 associations in patients with CFS}

Table 1 shows the allele frequencies of HLA-DRBI in the patient group and controls. Initial $\chi^{2}$ analysis revealed that the frequency distribution of HLA-DRBl alleles was not significantly different between the CFS group and the controls (likelihood ratio: $\chi^{2}=17.5$; degrees of freedom, $10 ; \mathrm{p}=0.1)$. However, examination of the adjusted residuals indicated that the frequency of the HLA-DRB1* 11 allele was significantly different to controls and made the largest contribution to the $\chi^{2}$ value. Further analysis by $2 \times 2$ contingency tables revealed a decreased frequency of HLA$\mathrm{DRBI}^{*} 11$ alleles in patients with CFS compared with controls, although this was only weakly significant (5.1\% v 12.5\%; OR, $0.4 ; 95 \% \mathrm{CI}, 0.12$ to $1.08 ; \mathrm{p}=0.046)$.

\section{HLA-DQA 1 and HLA-DQB 1 associations in patients with CFS}

The overall frequency distribution of the HLA-DQAl and HLA-DQB1 alleles in the CFS group was not significantly different from the controls (table 1). However, examination of adjusted residuals indicated that there were differences in the frequency of the HLA-DQA1 ${ }^{*} 01$ and HLA-DQB ${ }^{*} 06$ alleles between the patients and controls. Analysis by $2 \times 2$ contingency tables revealed an increased frequency of HLADQAl ${ }^{*} 01$ in patients with CFS $(51.0 \% v 35 \%$; OR, $1.93 ; 95 \%$ CI, 1.2 to $3.3 ; \mathrm{p}=0.008)$. HLA-DQBI ${ }^{*} 06$ was also increased in the patients with CFS $(30.2 \% v 20.0 \%$; OR, 1.73 ; $95 \%$ CI, 0.96 to 3.1), although this was on the borderline of significance $(\mathrm{p}=0.052)$.

The HLA-DRBl, HLA-DQAl, and HLA-DQBl associations need to be treated with caution because they have not been corrected for multiple comparisons. If corrected for all 22 possible comparisons then significance was lost. However, a less conservative approach of correcting for multiple comparisons at each multiallelic locus (at HLA-DRBl, HLA-DQAl, and HLA-DQB1 separately), suggests a possible association of HLA-DQAl ${ }^{*} 01(\mathrm{p}=0.04)$ with CFS.

\section{Primary HLA class II association with CFS}

HLA-DQAl ${ }^{*} 0101$ alleles are in strong linkage disequilibrium with HLA-DQB ${ }^{*} 05$ and HLA-DRB ${ }^{*} 01$ alleles, whereas HLADQA1*0102/0103 alleles are in linkage disequilibrium with HLA-DQB1 ${ }^{*} 06$ and HLA-DRB1*15/16 alleles. Therefore, we

Table 2 Multivariate logistic regression analysis to demonstrate the association of HLA-DQA $1{ }^{*} 01$ with CFS, independent of $\mathrm{HLA}-\mathrm{DRB} 1{ }^{*} 01$ and $\mathrm{HLA}-\mathrm{DQB} 1{ }^{*} 05$

\begin{tabular}{lrlll}
\hline & $\begin{array}{c}\text { Regression } \\
\text { coefficient }\end{array}$ & SE & OR $(95 \% \mathrm{CI})$ & p Value \\
\hline Constant & -1.435 & 0.352 & & \\
DQA1 01 & 0.914 & 0.456 & $2.49(1.02$ to 6.1$)$ & 0.04 \\
DQB 1*05 & 0.234 & 0.613 & $1.26(0.4$ to 4.2$)$ & 0.7 \\
$\mathrm{DRB}^{*} 01$ & 0.036 & 0.647 & $1.04(0.3$ to 3.7$)$ & 0.9 \\
\hline
\end{tabular}

Multivariate logistic regression analysis with presence or absence of CFS as the dependent variable. All independent variables were adjusted for each other in the model. The $p$ values represent the significance of each variable compared with individuals negative for that variable.

$\mathrm{CFS}$, chronic fatigue syndrome; $\mathrm{Cl}$, confidence interval; OR, odds ratio. 
Table 3 Multivariate logistic regression analysis to demonstrate the association of $\mathrm{HLA}-\mathrm{DQA}{ }^{*} 01$ with CFS, independent of $\mathrm{HLA}-\mathrm{DRB} 1{ }^{*} 15 / 16$ and $\mathrm{HLA}-\mathrm{DQB} 1{ }^{*} 06$

\begin{tabular}{lrlll}
\hline & $\begin{array}{c}\text { Regression } \\
\text { coefficient }\end{array}$ & SE & OR (95\% Cl) & p Value \\
\hline Constant & -1.435 & 0.352 & & \\
DQA1*01 & 1.004 & 0.492 & $2.73(1.04$ to 7.2$)$ & 0.04 \\
DQB1*06 & 0.208 & 0.486 & $1.23(0.5$ to 3.2$)$ & 0.7 \\
DRB1*15/16 & -0.257 & 0.485 & $0.77(0.3$ to 2.0$)$ & 0.6 \\
\hline
\end{tabular}

Multivariate logistic regression analysis with presence or absence of CFS as the dependent variable. All independent variables were adjusted for each other in the model. The $p$ values represent the significance of each variable compared with individuals negative for that variable.

$\mathrm{CFS}$, chronic fatigue syndrome; $\mathrm{Cl}$, confidence interval; $\mathrm{OR}$, odds ratio

investigated whether HLA-DQAl ${ }^{*} 01$ might form part of an extended haplotype with other class II HLA alleles, thereby explaining the association with CFS. In the absence of family data we could not assign definitive haplotypes to individual patients. Instead, we carried out logistic regression analyses in which combinations of phenotypes were included together as one variable (for example, HLA-DRBI*01, HLA-DQAl*01, and HLA-DQBI*05) for comparison between patients with CFS and controls. Any individual not carrying this combination was considered negative. No significant differences were found between patients and controls for any combination of HLA-DRB1, HLA-DQAl, and HLA-DQBl phenotypes.

We next carried out multivariate logistic regression analyses to investigate whether the HLA-DQAl*01 association was independent of HLA-DQBI and HLA-DRBI. Individual HLA-DRB1, HLA-DQAl, and HLA-DQBl phenotypes were included as separate independent variables in the same model. Inclusion of all these variables suggested that the primary association with CFS was with HLA-DQAl ${ }^{*} 01$, and that this was independent of the HLA-DRBI and HLADQB1 alleles. Table 2 shows that HLA-DQAl ${ }^{*} 01$ is associated with CFS independent of HLA-DRBI ${ }^{*} 01$ and HLA-DQB1 ${ }^{*} 05$. A similar result was found in a regression model containing HLA-DQAl*01, HLA-DRB1*15/16, and HLA-DQBI*06 (table 3 ).

Twenty five of the patients with CFS suffered from depression. Within the CFS group, there were no HLA class II associations with depression versus non-depression.

\section{DISCUSSION}

A genetic component to the development of CFS has been suggested by various twin studies. ${ }^{14-16}$ Immune dysfunction studies $^{9}{ }^{102}$ highlighted cytokine deregulation and immune cell disruption ${ }^{27}$ as important features in the pathophysiology of the disease. In our current study, we show an increase in the expression of HLA DQA ${ }^{*} 01$ in patients with CFS. There was also a marginal increase in the expression of HLA$\mathrm{DQBI}^{*} 06$, although the association with CFS disappeared after inclusion of HLA-DQBI ${ }^{*} 06$ as an independent variable in the same logistic regression model as HLA-DQA*01. This suggests that HLA-DQAl*01 may be the primary association with CFS. We also found decreased expression of HLA$\mathrm{DRBI}^{*} 11$ in CFS, although this association was weak and disappeared after correction for all possible comparisons of HLA-DRBI alleles between patients and controls.

Specific HLA-DQ polymorphisms have been associated with various diseases including diabetes and coeliac disease. $^{28}{ }^{29}$ In previous work, Keller et al found a possible association of HLA-DQ3 with chronic fatigue syndrome, ${ }^{7}$ but HLA-DQ3 is a broad definition based on serological typing, so any association with disease could not be assigned to specific HLA-DQ alleles. The HLA-DQA1 ${ }^{*} 01$ allele has been associated with few disease conditions, although associations have been found with tubulointerstitial nephritis and uveitis syndrome, ${ }^{30}$ and hyper-response to measles vaccine. ${ }^{31}$

\section{"Although our results will need independent replication, they do provide possible target HLA alleles to investigate further for association with chronic fatigue syndrome"}

Underhill et al found no association between HLA alleles and CFS. ${ }^{19}$ However, they did not examine the frequency of the HLA-DQAl alleles, so it is not possible to make a direct comparison of results. Interestingly, in their study the combined frequency of the HLA-DQBI*0601 and HLADQB1*0602 phenotypes was significantly higher in the patients than in the controls $(35 \% \vee 21 \%$; $p=0.045)$, if correction for multiple comparisons was omitted. Although not commented upon in the Underhill study, these data are consistent with the marginal increase in HLA-DQB ${ }^{*} 06$ allele frequency seen in our study, and may be indicative of an increased frequency of HLA-DQAl ${ }^{*} 01$ alleles in linkage disequilibrium. The patients studied by Underhill et al came from the same clinical service as in our present study, although they were different patients. ${ }^{19}$ Therefore, any differences between the two studies are unlikely to be explained by selection bias.

HLA-DQBI*06 forms the largest group of HLA-DQBI molecules, ${ }^{32}$ and has been associated with several diseases, including narcolepsy ${ }^{33}$ and multiple sclerosis. ${ }^{34}$ Interestingly, it has been suggested that narcolepsy, which is strongly associated with HLA-DQB1*0602, may need to be excluded in the differential diagnosis of CFS. ${ }^{35}$ The marginal increase in HLA-DQB1*06 seen in our study will need to be investigated further. The same is true for the decreased expression of HLA-DRB1* 11 .

There may be several reasons for the inconsistency seen in studies of HLA molecules and CFS. Early studies used serological typing, which is unable to discriminate between many of the alleles identified by the sequence specific primer/ polymerase chain reaction technique used here. Even so, additional subtyping of HLA-DQAl ${ }^{*} 01$ and HLA-DQBI*06 alleles will be necessary to identify which particular subtypes are important. A second major problem is that the few studies that have been carried out have used relatively small numbers of patients with CFS, and may lack sufficient power to show significant differences. Our current study also suffers from this caveat and, although differences between patients and controls were detected, the results need to be treated with caution. In particular, the need for multiple testing at multiallelic loci is a major problem in this type of study, especially when the number of available cases is low. Although our results will need independent replication, they do provide possible target HLA alleles to investigate further for association with CFS.

In conclusion, the current available data on immune cell and cytokine deregulation in CFS are consistent with an immunomodulatory role for the HLA system in this disease.

\section{Take home messages}

- Our results suggest an association between chronic fatigue syndrome (CFS) and the HLA class II region, primarily the HLA-DQA $1{ }^{*} 01$ allele

- However, because of the strong linkage disequilibrium between genes in this region, an association with other genes within the HLA locus cannot be ruled out

- Further studies are needed to clarify the situation 
The results of our study provide provisional evidence for an association of CFS with the HLA class II region, and suggest that this may be primarily with the HLA-DQAl*01 allele. However, because of the strong linkage disequilibrium between genes in this region, an association with other genes within the HLA locus cannot be ruled out.

\section{ACKNOWLEDGEMENTS}

This research was funded by the CFS Research Foundation, Hertfordshire, UK and the Royal Brompton and Harefield NHS Trust. We also thank D Blair, who was funded by the Joint Research Council of King's College Hospital and GKT School of Medicine, for help in subject recruitment and sample collection.

\section{Authors' affiliations}

E L Fritz, National Heart and Lung Institute, Imperial College London SW3 6NP, UK

J Smith, Tissue Typing Laboratory, Harefield Hospital, Middlesex UB9 6JH, UK

J R Kerr, Department of Paediatric Infectious Diseases, St Mary's Campus, Imperial College London UB9 6JH, UK

A J Cleare, S Wessely, Institute of Psychiatry and Maudsley Hospital, Guy's, King's and St Thomas's School of Medicine, King's Campus, PO Box 065, 103 Denmark Hill, London SE5 8AZ, UK

D L Mattey, Staffordshire Rheumatology Centre, University Hospital of North Staffordshire, Stoke on Trent ST6 7AG, UK

\section{REFERENCES}

1 Evengard B, Klimas N. Chronic fatigue syndrome: probable pathogenesis and possible treatments. Drugs 2002;62:2433-46.

2 Buchwald D, Umali P, Umali J, et al. Chronic fatigue and the chronic fatigue syndrome: prevalence in a Pacific northwest health care system. Ann Intern Med 1995; 123:81-8.

3 Georgiades E, Behan W, Kilduff L, et al. Chronic fatigue syndrome: new evidence for a central fatigue disorder. Clin Sci (Lond) 2003;105:213-18.

4 Fukuda K, Straus S, Hickie I, and The International Chronic Fatigue Syndrome Study Group, et al. The chronic fatigue syndrome: a comprehensive approach to its definition and study. Ann Intern Med 1994;121:953-9.

5 Chia J, Chia A. Diverse etiologies for chronic fatigue syndrome. Clin Infect Dis 2003;36:671-2.

6 Kerr J, Barah T, Mattey D, et al. Circulating tumour necrosis factor- $\alpha$ and interferon- $\gamma$ are detected during acute and convalescent parvovirus B19 infection and are associated with prolonged and chronic fatigue. J Gen Virol 2001;82:3011-19.

7 Keller R, Lane J, Klimas N, et al. Association between HLA class II antigens and the chronic fatigue immune dysfunction. Clin Infect Dis 1994;18(suppl 1):S154-6.

8 Hassan I, Bannister B, Akbar A, et al. A study of the immunology of the chronic fatigue syndrome: correlation of immunologic parameters to the health dysfunction. Clin Immunol Immunopathol 1998;87:60-7.

9 Patarca R. Cytokines and chronic fatigue syndrome. Ann NY Acad Sci 2001;993:185-200.

10 Patarca-Montero R, Klimas N, Fletcher M. Immunotherapy of chronic fatigue syndrome: therapeutic interventions aimed at modulating the Th1/Th2 cytokine expression balance. J Chronic Fatigue Syndr 2001;8:3-37.
11 Fletcher M, Maher K, Klimas N. Natural killer cell function in chronic fatigue syndrome. Clinical and Applied Immunology Reviews 2002;2:129-39.

12 Natelson BH, La Manca JJ, Denny TH, et al. Immunologic parameters in chronic fatigue syndrome, major depression, and multiple sclerosis. Am J Med 1998; 105:43S-95.

13 Lyall M, Wessely S, Peakman M. A systematic review and critical evaluation of the immunology of chronic fatigue syndrome. J Psychosom Res 2003:55:79-90.

14 Hickie IB, Bansal AS, Kirk KM, et al. A twin study of the etiology of prolonged fatigue and immune activation. Twin Res $2001 ; 4: 94-102$.

15 Buchwald D, Herell R, Ashton S, et al. A twin study of chronic fatigue. Psychosom Med 2001;63:936-43.

16 Koelle DM, Barcy S, Huang ML, et al. Markers of viral infection in monozygotic twins discordant for chronic fatigue syndrome. Clin Infect Dis 2002;35:518-25.

17 Sabath DE, Barcy S, Koelle DM, et al. Cellular immunity in monozygotic twins discordant for chronic fatigue syndrome. J Infect Dis 2002;185:828-32.

18 Middleton D, Savage D, Smith D. No association of HLA class II antigens in chronic fatigue syndrome. Dis Markers 1991;9:47-9.

19 Underhill JA, Mahalingam M, Peakman M, et al. Lack of association between HLA genotype and chronic fatigue syndrome. Eur J Immunogenet $2001 ; 28: 425-8$.

20 Fitzgibbon $\mathbf{E}$, Whelto $\mathrm{H}$, Smith T. HLA DR class II antigens and post viral fatigue syndrome [abstract]. J Chronic Fatigue Syndr 1996;2:166.

21 Logean A, Rognan D. Recovery of known T-cell epitopes by computational scanning of a viral genome. J Comput Aided Mol Des 2002;16:229-43.

22 Vukmanović S, Neubert T, Santori F. Could TCR antagonism explain associations between MHC genes and disease? Trends Mol Med 2003;9:139-46.

23 Vollmer-Conna U, Lloyd A, Hickie I, et al. Chronic fatigue syndrome: an immunological perspective. Aust N Z J Psychiatry 1998;32:523.

24 Nachum F. Is interferon- $\alpha$ a neuromodulator? Brain Res Rev 1998;26:1-15.

25 Kerr J, Mattey D, Thomson W, et al. Association of symptomatic acute parvovirus B19 infection with human leukocyte antigen class I and II alleles. $J$ Infect Dis 2002;186:447-52.

26 Sharpe M, Chalder T, Palmer I, et al. Chronic fatigue syndrome. A practical guide to assessment and management. Gen Hosp Psychiatry 1997; 19:185-99

27 Whiteside T, Friberg D. Natural killer cells and natural killer cell activity in chronic fatigue syndrome. Am J Med 1998;105(3A):27S-34S

28 Karell K, Louka A, Moodie S, et al. HLA types in celiac disease patients not carrying the $\mathrm{DQA} 1{ }^{*} 05-\mathrm{DQB} 1{ }^{*} 02(\mathrm{DQ} 2)$ heterodimer: results from the European genetics cluster on celiac disease. Hum Immunol 2003;64:469-77.

29 Heward J, Mijovic C, Kelly M, et al. HLA-DQ and DRB1 polymorphism and susceptibility to type 1 diabetes in Jamaica. Eur J Immunogenet 2002;29:47-52.

30 Levinson RD, Park MS, Rikkers SM, et al. Strong associations between specific HLA-DQ and HLA-DR alleles and the tubulointerstitial nephritis and uveitis syndrome. Invest Ophthalmol Vis Sci 2003;44:653-7.

31 Hayney MS, Poland GA, Jacobson RM, et al. Relationship of HLA-DQA1 alleles and humoral antibody following measles vaccination. Int $J$ Infect Dis 1998:2:143-6.

32 Casamitiana N, Gil J, Campos E, et al. Identification of a novel HLA-DQB1 *06 allele, DQB 1*0618. Tissue Antigens 2001;58:269-71.

33 Chabas D, Taheri S, Renier C, et al. The genetics of narcolepsy. Annu Rev Genomics Hum Genet 2003;4:459-83.

34 Giordano M, D'Alfonso S, Momigliano-Richiardi P. Genetics of multiple sclerosis: linkage and association studies. Am J Pharmacogenomics 2002;2:37-58.

35 Ambrogetti A, Olson LG. Consideration of narcolepsy in the differential diagnosis of chronic fatigue syndrome. Med J Aust 1994;160:426-9. 